\title{
RE-ENTRY TRAJECTORY PLANNING FOR A REUSABLE LAUNCH VEHICLE
}

\author{
Kenneth D. Mease* \\ P. Teufel ${ }^{\dagger}$ H. Schönenberger $†$ D. T. Chen $;$ S. Bharadwaj ${ }^{\ddagger}$ \\ Department of Mechanical and Aerospace Engineering \\ University of California, Irvine, CA 92697
}

\begin{abstract}
The Shuttle's two-dimensional entry trajectory planning method is extended to threedimensions. Both angle of attack and angle of bank variations are used to control the entry trajectory. The trajectory planning is done with a third-order system of differential equations using the drag and lateral accelerations as intermediate controls. The reducedorder planning problem is solved as an optimal control problem. The state and control variables not involved in the planning are computed in a second step.
\end{abstract}

\section{Introduction}

A new generation of reusable launch vehicles and reusable orbit transfer vehicles are being developed. It seems likely that the entry guidance requirements for a reusable launch vehicle will be, as they are for the Shuttle, to steer the vehicle on a feasible trajectory - a trajectory within the entry corridor, defined by heating, acceleration, dynamic pressure and controllability limits - that achieves the specified target condition within the specified error margin. The Shuttle descent trajectory planning and guidance is composed of an entry phase and a terminal area energy management (TAEM) phase. The specified target condition for the entry phase is a longitude, latitude point at a certain Mach number. Achieving this target condition with sufficient accuracy (about two nautical miles) ensures a successful TAEM. The entry capability of reusable launch vehicles (RLVs) and orbital transfer vehicles (OTVs) would be significantly enhanced relative to that afforded by Shuttle-type entry guidance, if the following entry planning capabilities can be attained.

1. Trajectory planning to meet both downrange and crossrange objectives precisely. The reference trajectory is planned so as to reach the desired target condition, and also to pass over desired intermediate points (i.e. waypoints). The latter capability may be required, for example, to avoid heavily populated areas during overland flight. In contrast the reference trajectory planning for the Shuttle entry guidance only considers the downrange objectives; crossrange objectives are handled less precisely through bank reversal logic.

${ }^{*}$ Professor; Associate Fellow AIAA

†Visiting Student, University of Stuttgart

¥ Graduate Research Assistant

Copyright (C) 1999 by the authors. Published by the American Institute of Aeronautics and Astronautics, Inc. with permission.
2. Use of both angle of attack and angle of bank as commanded variables to achieve guidance objectives. The Shuttle entry guidance fixes the angle of attack profile for trajectory planning and only uses minor modifications in tracking to smooth out transient behavior during bank reversals. While this simplifies the guidance logic, it limits entry capability. It is true that heating constraints dictate high angle of attack early in the entry and that it is desired to be on the front side of the $L / D$ curve at the initiation of the terminal area energy management phase; nonetheless, these requirements still leave freedom in the angle of attack profile that can be used to enlarge the landing footprint, increase guidance accuracy, and minimize bank reversals.

3. Onboard rapid trajectory planning to achieve greater capability and autonomy for both nominal and abort missions. With near real-time onboard trajectory planning, an RLV can be much more responsive to trajectory dispersions and mission changes. Such responsiveness will enhance performance and safety.

In this paper we develop a trajectory planning approach that achieves the desired capabilities. Our trajectory planning approach is a direct generalization of the trajectory planning concept for the Shuttle. ${ }^{1}$

\section{Entry Planning Problem}

The state space for the translational motion (i.e., the position-velocity space) is 6-dimensional. Since we will model the entry dynamics as time-independent, we can consider 5 state variables to be functions of a sixth state variable, if this sixth state variable is strictly monotonic along the trajectories of interest. 
We shall consider 5 state variables as functions of the strictly decreasing energy $E$ defined by

$$
E=\frac{1}{2} V^{2}-\left(\frac{\mu}{r}-\frac{\mu}{r_{s}}\right)
$$

where $V$ is the velocity magnitude, $r$ and $r_{s}$ are the radial distances from the planet center to the vehicle center of mass and the planet surface, respectively, and $\mu$ is the gravitational constant. This is an appropriate formulation for the entry problem since the terminal conditions are given at an energy value, whereas time plays no role.

Denoting $d(\cdot) / d E$ by $(\cdot)^{\prime}$ and using $(\cdot)^{\prime}=$ $(1 / \dot{E}) d(\cdot) / d t$, the translational equations of motion ${ }^{8}$ for the center of mass of an unpowered vehicle of constant mass flying over a non-rotating spherical planet with a stationary atmosphere take the form

$$
\begin{aligned}
\theta^{\prime} & =-\frac{\cos \gamma \sin \chi}{r \cos \phi}\left(\frac{1}{D}\right) \\
\phi^{\prime} & =-\frac{\cos \gamma \cos \chi}{r}\left(\frac{1}{D}\right) \\
\chi^{\prime} & =-\frac{\cos \gamma \sin \chi \tan \phi}{r}\left(\frac{1}{D}\right)+\frac{1}{V^{2}}\left(\frac{L \sin \sigma}{D}\right) \\
r^{\prime} & =-\sin \gamma\left(\frac{1}{D}\right) \\
\gamma^{\prime} & =\left(g-\frac{V^{2}}{r}\right) \frac{\cos \gamma}{V^{2}}\left(\frac{1}{D}\right)-\frac{1}{V^{2}}\left(\frac{L}{D} \cos \sigma\right)
\end{aligned}
$$

where $\theta$ is the longitude, $\phi$ is the latitude, $\gamma$ is the flight path angle, and $\chi$ is the heading angle with $\chi=0$ corresponding to north. The bank angle $\sigma$ is defined such that the lift vector is in the vertical plane at zero bank. The acceleration due to gravity is $g=\mu / r^{2}$. $L$ and $D$ represent the lift and drag accelerations (specific forces) and are given by

$$
\begin{aligned}
L & =\frac{1}{2} \rho(r) V^{2} \cdot \frac{S}{m} \cdot C_{L}(\alpha, V, r) \\
D & =\frac{1}{2} \rho(r) V^{2} \cdot \frac{S}{m} \cdot C_{D}(\alpha, V, r)
\end{aligned}
$$

where $\rho(r)$ is the density as a function of the altitude, $C_{L}(\alpha, V, r)$ and $C_{D}(\alpha, V, r)$ are the lift and drag coefficients, $S$ is the reference area, and $m$ is the vehicle mass. The five state variables take values in the ranges: $0 \leq \theta<2 \pi,-\pi / 2<\phi \leq \pi / 2,-\pi<\chi \leq \pi$, $r \geq r_{s}$ and $-\pi<\gamma<\pi$.

\section{Vehicle and Control Constraints}

The vehicle constraints on the maximum dynamic pressure, aerodynamic acceleration, and heating rate are given by

$$
\begin{gathered}
q=\frac{1}{2} \rho V^{2} \leq q_{\max } \\
A=\left(D^{2}+L^{2}\right)^{1 / 2}=D(1+L / D)^{1 / 2} \leq A_{\max } \\
\dot{Q}=c \sqrt{\rho} V^{3} \leq \dot{Q}_{\max }
\end{gathered}
$$

The angle of attack $\alpha$ and the angle of bank $\sigma$ are taken to be the controls. There may be restrictions on the values of both controls. In this paper we only consider energy-dependent bounds on $\alpha$; namely, we require that $\alpha(E) \in\left[\alpha_{\min }(E), \alpha_{\max }(E)\right]$.

\section{Problem Statement}

To simplify notation, let $x=(\theta, \phi, \chi, r, \gamma)$ denote the state. The entry dynamics are expressed concisely as $x^{\prime}=f(x, \alpha, \sigma)$. The target longitude $\theta_{f}$ and latitude $\phi_{f}$ are given at a specified final energy $E_{f}$; these specifications are represented as terminal equality constraints $\Psi\left(x\left(E_{f}\right)\right)=\left(\theta\left(E_{f}\right)-\theta_{f}, \phi\left(E_{f}\right)-\phi_{f}\right)^{T}=$ $(0,0)^{T}$. We note that the final heading angle could be specified also.

The entry trajectory planning problem is: given the state $x\left(E_{0}\right)$ at an initial energy $E_{0}$, the terminal constraints, and the vehicle constraints, determine feasible controls $(\alpha(E), \sigma(E))$ on the interval $\left[E_{0}, E_{f}\right]$. Feasible means that the state trajectory and the controls satisfy the boundary conditions and the vehicle and control constraints.

\section{Existence and Uniqueness of a Solution}

For each initial condition, there is a 5-dimensional reachable set $\mathcal{R}\left(E_{f} ; x\left(E_{0}\right)\right)$ of terminal states contained in the 5 -dimensional terminal energy slice, defined by $E=E_{f}$, of the 6-dimensional state space. The terminal conditions are two independent constraints on the final state and define a 3 -dimensional terminal manifold in the terminal energy slice of the state space. If the intersection of the terminal manifold and the reachable set is empty, then there is no solution to the entry problem. If the intersection is non-empty, then a solution exists. In this case, the solution typically will not be unique, unless the intersection is a single point on the boundary of the reachable set, e.g., the maximum range solution.

\section{Soft Constraints}

In cases where there are multiple solutions to the entry problem, one can impose additional specifications to distinguish and choose between them. At each energy and at each magnitude of the bank angle, there is a minimum vertical component of lift

$$
(L \cos \sigma)_{\text {min }}=\left(g-\frac{V^{2}}{r}\right)
$$

dictated by the equilibrium glide boundary; below this minimum vertical lift the vehicle cannot generate enough lift force to balance the effective weight. 
For each value of $\alpha$, there is a maximum altitude that corresponds to the equilibrium glide boundary. The vehicle does not have enough control authority to sustain level flight above this altitude, although transient excursions are possible. By staying within the equilibrium glide boundary, there is excess vertical lift capability to compensate for trajectory dispersions.

In addition to keeping the heating rate $\dot{Q}$ below a specified upper limit it is desirable to minimize the heat load

$$
Q=\int_{E_{0}}^{E_{f}} \dot{Q}\left(\frac{d E}{d t}\right)^{-1} d E
$$

This is accomplished by flying on the active vehicle constraint boundary - dynamic pressure, heating rate, or aerodynamic acceleration - for much of the trajectory, which shortens the flight time. ${ }^{4}$ On the other hand, to accommodate dispersions it is desirable for the trajectory to be away from the constraint boundaries. The most desirable entry trajectory is some compromise between minimizing the heat load and leaving margin for dispersions.

\section{Shuttle 2D Trajectory Planning}

The onboard entry trajectory planning for the U.S. Space Shuttle Orbiters ${ }^{1}$ considers only the longitudinal motion. Consider flight in the equatorial plane so that $\chi=\pi$ and $\phi=0$. Assuming that $\cos \gamma=1$ and that $r(E)=\hat{r}(E)$, where $\hat{r}$ is an estimate of the radial position profile, we have

$$
\theta^{\prime}=-\frac{1}{\hat{r}}\left(\frac{1}{D}\right)
$$

For the Shuttle the bank angle is the primary entry trajectory control; the angle of attack assumes a fixed profile for planning. For planning purposes, the drag acceleration $D$ rather than the bank angle is considered to be the control. Under the assumptions, the longitude depends only on the drag profile, i.e., the drag acceleration as a function of $E$. In fact, by using a piecewise quadratic function of $E$, the Shuttle trajectory planning is done analytically. The vehicle constraints can all be represented as drag acceleration constraints. An entry corridor can be plotted in the drag versus energy plane. At each energy, there is a maximum drag dictated by the active vehicle constraint. There is also a minimum drag dictated by the zero bank equilibrium glide inequality

$$
L \geq\left(g-\frac{V^{2}}{r}\right)
$$

With $\alpha$ and $r$ given as functions of $E$, there is a unique value of $L / D$ for each value of $E$. Thus we have

$$
D_{\min }=L_{\min }\left(\frac{L}{D}\right)^{-1}=\left(g-\frac{V^{2}}{r}\right)\left(\frac{L}{D}\right)^{-1}
$$

Once the drag profile has been determined, the other trajectory and control information can be computed. The first and second derivatives of the drag acceleration with respect to energy provide algebraic relations satisfied by the state and control variables. At each energy, with the angle of attack given, $r$ can be extracted from the drag model. The $D^{\prime}$ and $D^{\prime \prime}$ equations can then be solved for $\gamma$ and $(L / D) \cos \sigma$. The magnitude of $\sigma$ can be extracted from $(L / D) \cos \sigma$.

\section{Reduced-Order 3D Trajectory Planning}

Reduced-order system

Under the same approximations

$$
\begin{gathered}
r(E)=\hat{r}(E) \\
\cos \gamma=1
\end{gathered}
$$

we propose a similar approach for trajectory planning in both the longitudinal and latitudinal dimensions, thus extending the Shuttle trajectory planning. At a minimum, we would need to employ the differential equations for longitude and latitude. In addition to drag, we could treat the heading angle $\chi$ as a control. However, it is not clear what values of $\chi$ would lead to a feasible entry trajectory. By including the differential equation for the heading angle, we can instead treat the lateral acceleration $L \sin \sigma$ as the second control. The drag and lateral accelerations are perhaps the most natural controls for generalizing the Shuttle trajectory planning. The control $(L / D) \sin \sigma$ is, however, a little more convenient and we shall use it. Thus the reduced-order system for trajectory planning is

$$
\begin{aligned}
\theta^{\prime} & =-\frac{\sin \chi}{\hat{r} \cos \phi}\left(\frac{1}{D}\right) \\
\phi^{\prime} & =-\frac{\cos \chi}{\hat{r}}\left(\frac{1}{D}\right) \\
\chi^{\prime} & =\frac{1}{V^{2}}\left(\frac{L \sin \sigma}{D}\right)-\frac{\sin \chi \tan \phi}{\hat{r}}\left(\frac{1}{D}\right)
\end{aligned}
$$

The reference radius need not be constant; it may be a function of $E$. It is important to emphasize that the approximations, Eqs. (10), are only used when evaluating $\theta^{\prime}, \phi^{\prime}$ and $\chi^{\prime}$. We have essentially defined the small parameter $\epsilon=(r-\hat{r}) / \hat{r}$, employed the expansion $r^{-1}=\hat{r}^{-1}(1+\epsilon+\ldots)$, and written the differential equations for the zeroth-order approximations to $\theta, \phi$ and $\chi$. Once $\theta, \phi, \chi, D$, and $(L / D) \sin \sigma$ are determined, an extraction algorithm, described later, will be used to determine the corresponding values of $r, \gamma$, $\alpha$ and $\sigma$. The extracted values of $r$ and $\gamma$ in general will not be $\hat{r}$ and 0 , respectively. 


\section{Admissible control set}

We need to define the admissible set of values of the intermediate controls $D$ and $(L / D) \sin \sigma$. The first step is to express the vehicle constraints as constraints on the drag acceleration. The dynamic pressure, aerodynamic acceleration, and heating rate constraints take the form

$$
\begin{gathered}
D \leq q_{\max } S C_{D}(\alpha, E, \hat{r}) \\
D \leq A_{\max }\left(1+\frac{L}{D}(\alpha, E, \hat{r})\right)^{-1 / 2} \\
D \leq \dot{Q}_{\max } \frac{C_{D} S}{2 m c^{2} V^{2(k-1)}}
\end{gathered}
$$

We approximate $r$ by $\hat{r}$ in these constraints. Then at each value of the energy in the range $\left[E_{0}, E_{f}\right]$, the drag constraints depend only on $\alpha$. We now describe the process for generating the admissible set of values of the controls $(D,(L / D) \sin \sigma)$ at each value of $E$. An example of the admissible set for a particular value of $\mathrm{E}$ is shown in Fig. 1.

For a given value of $\alpha \in\left[\alpha_{\min }, \alpha_{\max }\right]$, the maximum drag acceleration, $D_{\max }$, is the least upper bound dictated by the three drag constraints. For the same value of $\alpha$, we compute the lift-to-drag ratio $L / D$. Next we plot the $\left(D_{\min },(L / D) \sin \sigma\right)$ curve generated by different values of $\sigma$ according to

$$
\begin{gathered}
D_{\min }=\frac{g-V^{2} / \hat{r}}{(L / D) \cos \sigma} \\
\frac{L}{D} \sin \sigma=\frac{L}{D}\left(1-\cos ^{2} \sigma\right)^{1 / 2}
\end{gathered}
$$

The first equation is derived from the level-flight equilibrium glide condition. With $L / D$ fixed by the value of $\alpha$, specifying $\sigma$ determines the minimum value of $D$ at which the lift force will balance the effective weight, $m\left(g-V^{2} / r\right)$, and also determines the lateral acceleration $L \sin \sigma$, or equivalently, $(L / D) \sin \sigma$. We start with $\sigma=0$ and work up to the value of $\sigma$ for which $D_{\text {min }}=D_{\text {max }}$; larger values of $\sigma$ are not admissible. The curve for negative values of $\sigma$ is just the reflection about the $D$-axis of curve for positive $\sigma$. Repeating these calculations, for each value of $\alpha$, of $D_{\max }$ and the curves of $\left(D_{\min },(L / D) \sin \sigma\right)$, generates the admissible set for a given energy.

The maximum value of drag and the maximum value of $(L / D) \sin \sigma$ is calculated at a specific values of $\alpha$, the curve through these points is labeled "max drag curve". Observe that with decreasing values of $\alpha$ the range for $D$ and $L / D \sin \sigma$ is becoming smaller. In the example, $\alpha_{\min }$ is the $\alpha$ corresponding to $(L / D)_{\max }$. If $\alpha_{\min }$ was less than the values for $(L / D)_{\max }$, then the maximum $L / D$ point on the maximum drag curve would not correspond to $\alpha_{\min }$. The largest admissible set has a boundary on the maximum $L / D$ equilibrium glide curve, since the equilibrium glide curves moves inward for lower values of $\alpha$ on the front side of the $L / D$ curve. Repeating these calculations for each energy will generate the entire admissible set.

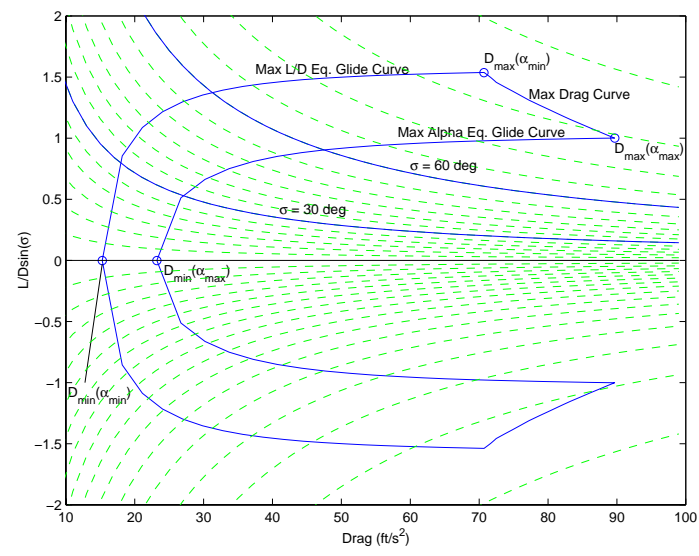

Fig. 1 Admissible Controls

\section{Optimal control formulation}

The re-entry trajectory planning problem will be solved as an optimal control problem of the following form: Minimize the performance index

$$
J=\Phi\left(x\left(E_{f}\right), E_{f}\right)+\int_{E_{0}}^{E_{f}} \mathcal{L}(x(E), u(E), v(E), E) d E
$$

subject to the following conditions:

- the differential constraints

- initial state conditions

$$
x(0)=x_{0}
$$

- the terminal state conditions

$$
\Psi\left(x\left(E_{f}\right), E_{f}\right)=0
$$

- the algebraic state and control constraints

$$
g(x(E), u(E), v(E)) \geq 0, \forall E \in\left[E_{0}, E_{f}\right]
$$

where $x=(\theta, \phi, \chi), u=\left(u_{1}, u_{2}\right)=(D, L / D \sin \sigma)$, and $v=\left(v_{1}, v_{2}\right)$. Along with the three reduced order equations, the two additional differential equations $u_{1}^{\prime}=v_{1}$ and $u_{2}^{\prime}=v_{2}$ are included in the differential constraints.

A variety of specific formulations are possible, depending on whether the various constraints are handled as soft (i.e, in the performance index) or hard (as equality or inequality constraints). A versatile performance index is defined by

$$
\begin{array}{cl}
\Phi\left(x\left(E_{f}\right)\right)= & a_{1}\left(\theta\left(E_{f}\right)-\theta_{f}\right)^{2}+a_{2}\left(\phi\left(E_{f}\right)\right. \\
& \left.-\phi_{f}\right)^{2}+a_{3}\left(\chi\left(E_{f}\right)-\chi_{f}\right)^{2} \\
\mathcal{L}(x(E), u(E), v(E), E)= & a_{4} \frac{V}{\sqrt{D}}+a_{5}\left(v_{1}(E)^{2}+v_{2}(E)^{2}\right) \\
& +a_{6}\left(b_{1} F_{1}^{2}+b_{2} \frac{1}{F_{2}}+b_{3} F_{3}\right)
\end{array}
$$


where $a_{1}$ through $a_{6}$ and $b_{1}$ through $b_{2}$ are nonnegative constants. The constants $a_{1}$ through $a_{3}$ are non-zero if the terminal conditions are treated as soft constraints rather than equality constraints. The constant $a_{4}$ is non-zero if the heat load is included in $J$. We note that $V / \sqrt{D}$ is proportional to $d Q / d E$. When $a_{5}$ is non-zero, the rates of change of $u_{1}$ and $u_{2}$ with respect to energy are penalized. The constant $a_{6}$ is non-zero when the condition that $\left(u_{1}, u_{2}\right)$ belong to the admissible control set is handled as a soft constraint. The functions $F_{1}, F_{2}$, and $F_{3}$ are representative of the maximum drag, $\alpha_{\max }$ equilibrium glide, and $(L / D)_{\max }$ equilibrium glide constraint curves of the admissible set and constitute the boundaries of the admissible set as shown in Fig. 2. One of these functions is equal to one when $\left(u_{1}, u_{2}\right)$ is on the boundary of the admissible set. The equations for $F_{1}, F_{2}$, and $F_{3}$ that approximate the boundaries of the admissible set are

$$
\begin{aligned}
F_{1}= & \frac{u_{2}(E)}{P_{1}\left(u_{1}\right)} \\
F_{2}= & \left(\frac{u_{1}(E)-P_{n}^{(3)}(E)}{P_{n}^{(1)}(E)-P_{n}^{(3)}(E)}\right)^{4}+\left(\frac{u_{1}(E)-P_{n}^{(3)}(E)}{P_{n}^{(1)}(E)-P_{n}^{(3)}(E)}\right)^{2} \\
& +\left(\frac{u_{2}(E)}{P_{n}^{(2)}(E)}\right)^{2}+\left(\frac{u_{2}(E)}{P_{n}^{(2)}(E)}\right)^{4} \\
F_{3}= & \left(\frac{u_{1}(E)-P_{n}^{(6)}(E)}{P_{n}^{(4)}(E)-P_{n}^{(6)}(E)}\right)^{4}+\left(\frac{u_{1}(E)-P_{n}^{(6)}(E)}{P_{n}^{(4)}(E)-P_{n}^{(6)}(E)}\right)^{2} \\
& +\left(\frac{u_{2}(E)}{P_{n}^{(5)}(E)}\right)^{2}+\left(\frac{u_{2}(E)}{P_{n}^{(5)}(E)}\right)^{4}
\end{aligned}
$$

where $P_{1}\left(u_{1}\right)$ is a linear fit of $u_{1}$ to $u_{2}$ along the max drag boundary. $P_{n}^{(1)}(E)$ through $P_{n}^{(6)}(E)$ are $\mathrm{n}^{\text {th }}$-order polynomials in $E$. $P_{n}^{(1)}(E), P_{n}^{(2)}(E)$, and $P_{n}^{(3)}(E)$ fit the minimum drag, maximum $(L / D) \sin \sigma$, and maximum drag occurring on the maximum alpha boundary. $P_{n}^{(4)}(E), P_{n}^{(5)}(E)$, and $P_{n}^{(6)}(E)$ fit the minimum drag, maximum $(L / D) \sin \sigma$, and maximum drag occurring on the $(L / D)_{\max }$ equilibrium glide boundary (see Fig. 2).

\section{Determining remaining states and controls}

We used a reduced-order model to simplify, and reduce the numerical sensitivity of, the trajectory computation. The reduced-order trajectory planning determines $\theta, \phi, \chi, D$, and $(L / D) \sin \sigma$ as functions of $E$. The remaining state and control variables can be determined from this information and the differential equations.

Differentiating the model for the drag acceleration, with respect to $E$, and neglecting derivatives of the drag coefficient, yields

$$
D^{\prime}=\frac{2 D}{V^{2}}+\left(\frac{2 g}{V^{2}}+\frac{1}{H}\right) \sin \gamma
$$

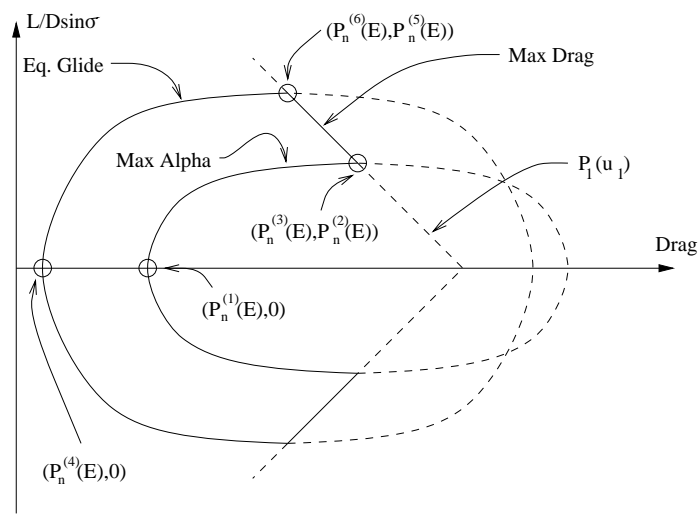

Fig. 2 Illustration of approximated sections for the admissible set boundaries.

Since the control rate $D^{\prime}$ is calculated in the optimal control problem, there is enough information with $r(E)=\hat{r}(E)$ to determine $\gamma(E)$. Using a backward finite difference on $\gamma$ to approximate $\gamma^{\prime}\left(E_{0}\right)$, we then have four algebraic equations from which to extract the four unknowns $r, \gamma, \alpha$, and $\sigma$ for the initial energy $E_{0}$. Future values of $r$ and $\gamma$ are determined by integrating the $r^{\prime}$ and $\gamma^{\prime}$ equations. The controls $\alpha$ and $\sigma$ are then calculated algebraically at each value of $E$.

\section{Results and Discussion}

As a first step in assessing the efficacy of our planning approach, we discuss and present results that were obtained by solving the optimal control problem with a general purpose optimization code: the Sparse Optimal Control Software (SOCS). ${ }^{10}$ We envision that a special purpose optimization algorithm would be used for the onboard implementation of our approach for faster computation and reduced code size. An accuracy assessment by comparison of our reduced-order planning solutions to full-order planning solutions is planned for future work. Here we shall describe our computational experience with different formulations of the optimal control problem and present a particular solution. We also present the results of applying the extraction algorithm to the same solution.

SOCS solves the optimal control problem using a direct transcription method. State and control variables are discretized to reduce the optimal control problem into a nonlinear programming problem. The solution to the sparse nonlinear program is then determined using sequential quadratic programming. SOCS is capable of generating a linear initial guess from given initial and final controls, or a subroutine can be written to generate an initial guess.

\section{Reduced-order planning algorithm}

The reduced-order planning algorithms for the various formulations were written primarily in FORTRAN. Performance testing and evaluation of the programs were done on a Pentium II $300 \mathrm{MHz} \mathrm{PC}$ with 
$64 \mathrm{Mb}$ of RAM running Windows 95. It was found that the most significant source of difficulty and computation time, for SOCS, was the enforcement of hard constraints for the admissible set. This formulation typically required 2-5 minutes of computation time which did not guarantee a feasible solution from SOCS. An addition of soft constraints on the admissible set to the performance index, improved the reliability of the program by keeping solutions away from the nonconvex $\alpha_{\max }$ equilibrium glide boundary. Once the hard constraints on the admissible set were removed, most of the reduced order trajectories required less than a minute of computation time with a crude initial guess. The program was capable of finding an optimal solution for a variety of initial and final conditions, performance index coefficients, initial guesses, and constraint formulations.

Of all the formulations tested, the formulation that gives the best results in terms of speed, reliability, and solution properties, uses hard constraints on $\theta\left(E_{f}\right)$, $\phi\left(E_{f}\right)$ and the two control rates, while the coefficients $a_{1}$ and $a_{2}$ of the general performance index Eq.(18) are set to zero. Due to the non-convex shape of the admissible set, the constraints on the admissible set are best replaced with soft constraints. Hard constraints on minimum alpha and maximum drag boundaries, can be enforced as redundant constraints to ensure that the trajectory does not violate those boundaries of the admissible set. The soft constraint on the admissible set and soft constraints on the control rates smoothes the control trajectory such that rapid variations from from one boundary of the admissible set to another do not occur. Having both hard and soft constraints on the control rate also minimizes the control power requirements and eliminates the occurrence of short peaks in control rates.

The reduced-order algorithm for 3D entry trajectory planning is applied to a vehicle model of a reusable second stage supplied by Boeing. The reusable second stage has a mass of $85000 \mathrm{lbs}$, reference area of 1300 $\mathrm{ft}^{2}$, maximum $C_{L}$ of 3.6 , and a maximum $L / D$ of 8.0 at an angle of attack of 10 degrees. To illustrate the capabilities of the trajectory planner, an example problem with initial conditions of zero degrees longitude, latitude, and heading and a final destination of 12 degrees longitude and 6 degrees latitude is used. The coefficients given for the performance index, Eqs.(14) and (18), are $a_{1}=a_{2}=a_{3}=0, a_{4}=5.55 \times 10^{-4}$, $a_{5}=3.85 \times 10^{-4}, a_{6}=0.385, b_{1}=0.7, b_{2}=4$, and $b_{3}=0.7$. The initial/final altitudes and velocities are set to $r_{i n i}=2.7 \times 10^{+5} \mathrm{ft}, r_{\text {final }}=9.0 \times 10^{+4}$ $\mathrm{ft}, V_{\text {ini }}=17500 \mathrm{ft} / \mathrm{s}$, and $V_{\text {final }}=3000 \mathrm{ft} / \mathrm{s}$ and linearly interpolated to obtain the reference radius and energy. The constraints on normal acceleration, dynamic pressure, and maximum angle of attack are set to $q_{\max }=600 \mathrm{lb} / \mathrm{ft}^{2}, A_{\max }=128 \mathrm{ft} / \mathrm{s}^{2}, \alpha_{\max }=40$ degrees, and $\alpha_{\min }=\alpha$ for $(L / D)_{\max }$.
Fig. 3 shows the admissible set of intermediate controls and the particular control trajectory for the reusable second stage vehicle model and specified boundary conditions. Note that the trajectory lies within the admissible set. Fig. 4 shows the trajectory

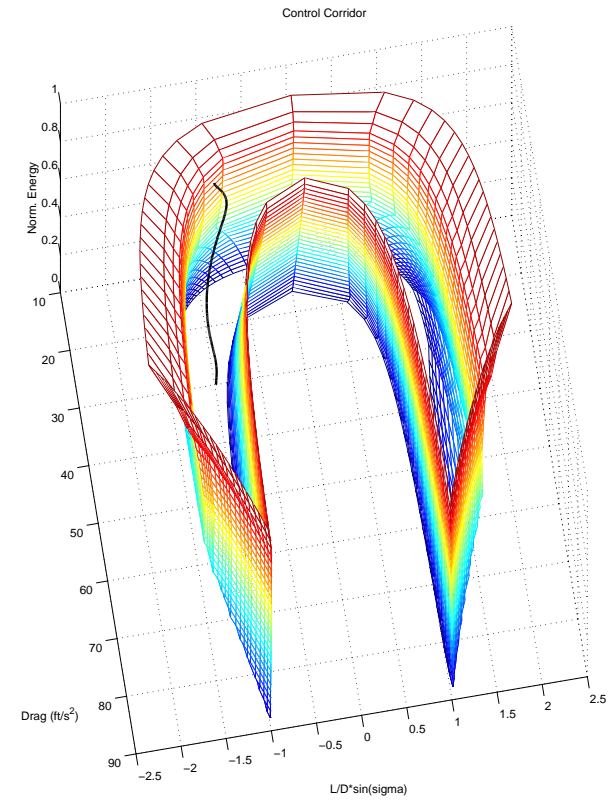

Fig. 3 Intermediate control trajectory within admissible set (boundaries of set shown).

in the longitude, latitude plane along with a plot of the heading angle as a function of normalized energy.
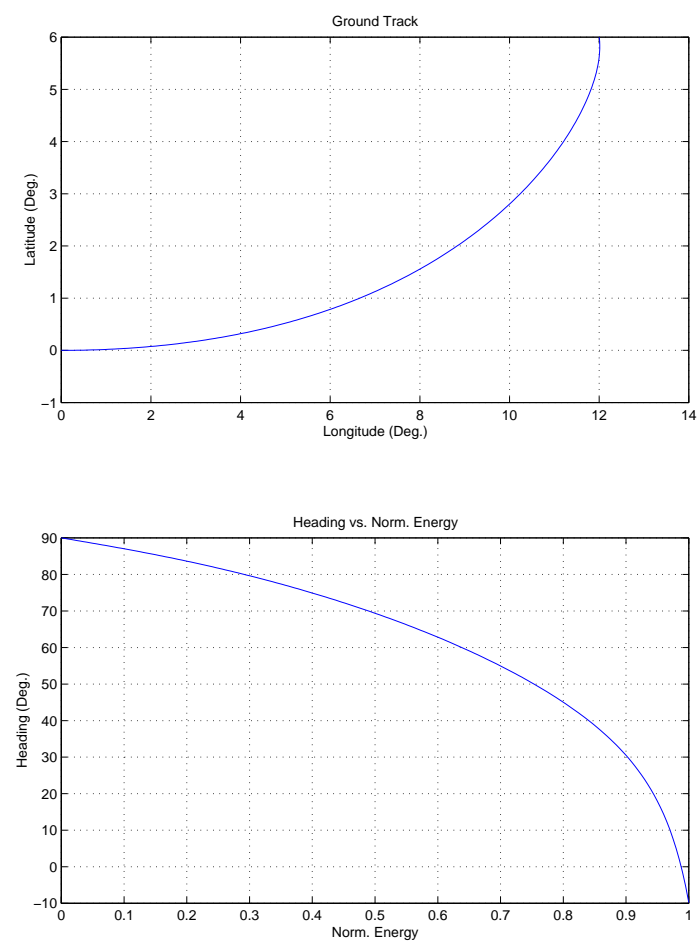

Fig. 4 Computed entry trajectory in longitude, latitude plane, and heading angle. 


\section{Extraction algorithm}

Once the optimal solution is obtained from the reduced-order planning the remaining states and controls have to be extracted. The complete state and control information is then used to perform an open loop simulation to determine the robustness and consistency of the extracted information with the reducedorder solution. The extraction algorithm described in the previous section was coded in MATLAB. Fig. 5 shows the state and control variables obtained from the extraction algorithm, given the reduced order solution from Fig. 3 and 4 . From the extracted gamma plot it can be seen that throughout the entire trajectory, the flight path angle remains relatively small and does not oscillate. The altitude plot shows that the profile follows a trajectory that is desirable for tracking because it does not oscillate much.

To perform the open-loop simulation, the controls along with an initial altitude and flight path angle from the extraction algorithm, are used to integrate the five equations of motion (2). A comparison of the differences between the downrange and crossrange trajectories from the simulation and the reduced-order planning are shown in Fig. 6. For the trajectory shown, the errors between the simulation and optimal solution for $\theta\left(E_{f}\right)$ and $\phi\left(E_{f}\right)$ are 0.136 and 0.071 degrees respectively. Plots of the two pseudo-controls $D$, and $(L / D) \sin \sigma$ from the optimal solution and simulation are shown in Fig. 7. From this plot it can be seen that the drag profile shows the most difference between the reduced-order solution and the profile obtained by integration. These consistency checks do not determine the accuracy of our solution. A comparison with fullorder solutions is needed for this.

\section{Conclusions}

The Shuttle's two-dimensional entry trajectory planning method has been extended to threedimensions. Both angle of attack and angle of bank variations are used to control the entry trajectory. The trajectory planning is performed with a thirdorder system of differential equations using the drag and lateral accelerations as intermediate controls. The reduced-order planning problem is solved as an optimal control problem. The state and control variables not involved in the planning are extracted from the planning solution in a second step.

\section{References}

${ }^{1}$ Harpold, J.D., and Graves, C.A., Jr., "Shuttle Entry Guidance," J. Astronautical Sciences, Vol. 27, No. 3, 1979, pp. 239-268.

${ }^{2}$ McEneaney, W.M. and Mease, K.D., "Error Analysis for a Guided Mars Landing," J. Astronautical Sciences, Vol. 39, No. 4, Oct.-Dec. 1991, pp. 423-445.

${ }^{3}$ Mease, K.D. and Kremer, J.-P., "Shuttle Entry Guidance Revisited Using Nonlinear Geometric Methods," J. Guidance, Control and Dynamics, Nov.-Dec. 1994, pp. 1350-1356.
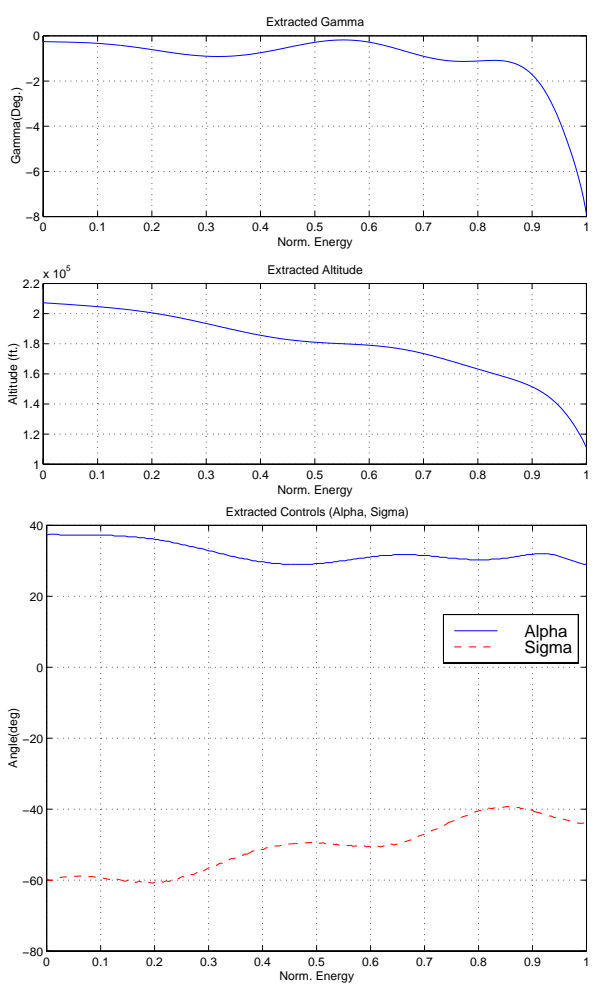

Fig. 5 Extracted states and controls $\gamma, r, \alpha$, and $\sigma$.

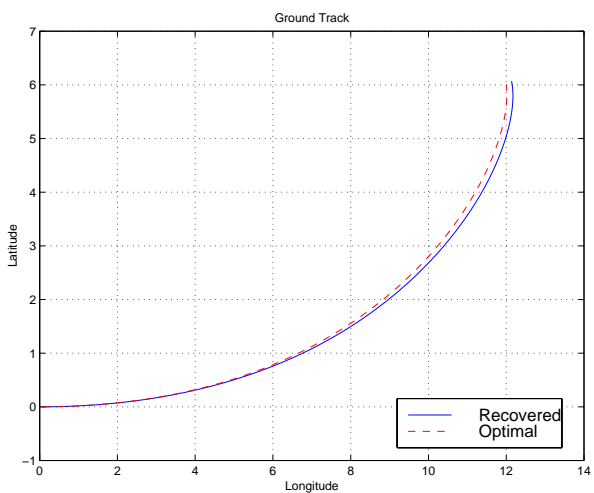

Fig. 6 Comparison of simulated and optimal ground track.

${ }^{4} \mathrm{Lu}$, P., "Entry Guidance and Trajectory Control for Reusable Launch Vehicle," J. Guidance, Control, and Dynamics, Jan.-Feb. 1997, pp. 143-149.

${ }^{5}$ Bharadwaj, S., Rao, A.V., and Mease, K.D., "Entry Trajectory Tracking Law via Feedback Linearization," J. Guidance, Control, and Dynamics, Sep.-Oct. 1998, pp. 726-732.

${ }^{6}$ Well, K.H. and Paus, M., "Optimal Ascent Guidance for a Hypersonic Vehicle," Paper 96-3901, AIAA GNCConference, San Diego, July 1996.

${ }^{7}$ Roenneke, A.J. and Markl, A., "Re-Entry Control to a Drag-vs-Energy Profile," J. Guidance, Control and Dynamics, Sep.-Oct. 1994, pp. 916-920.

${ }^{8}$ Vinh, N.X. Optimal Trajectories in Atmospheric Flight, Elsevier, New York, 1981.

${ }^{9}$ Stengel, R. F., "Optimal Guidance for the Space Shuttle Transition," J. Spacecraft, Vol. 11, No. 3, 1974, pp. 173-179.

${ }^{10}$ Betts, J. T. and Huffman, W. P., Sparse Optimal Con- 

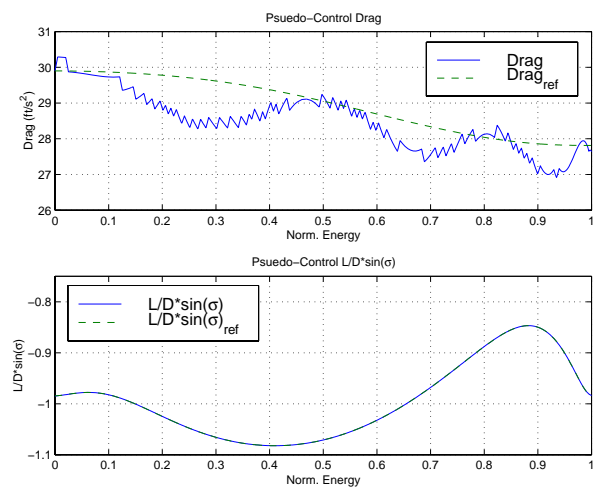

Fig. 7 Comparison of simulated and optimal pseudo-controls.

trol Software, The Boeing Co., Seattle, 1997. 